\title{
Data Available from \\ Birth and Death Registries \\ and Cancer Registries \\ in the United States
}

\author{
Becky L. Wallin, M.P.H.1,2 \\ Alan R. Houser, M.P.H. ${ }^{1}$ \\ Deane W. Merrill, Ph.D.1,3 \\ Steve Selvin, Ph.D.1,3
}

January 1994

1 Information and Computing Sciences Division, Lawrence Berkeley Laboratory, 1 Cyclotron Road, Berkeley, CA 94720.

2 Chicago Medical School, 3333 Green Bay Road, North Chicago, IL 60064.

3 Department of Biomedical and Environmental Health Sciences, University of California, Berkeley, CA 94720.

Address all correspondence to Alan R. Houser, Information and Computing Sciences Division, 50B2239, Lawrence Berkeley Laboratory, 1 Cyclotron Road, Berkeley, CA 94720. Fax: (510) 486-6363.

This work was supported by the Director, Office of Epidemiology and Health Surveillance; Office of Health; Office of Environment, Safety and Health; U.S. Department of Energy under Contract No. DEAC03-76-SF00098.

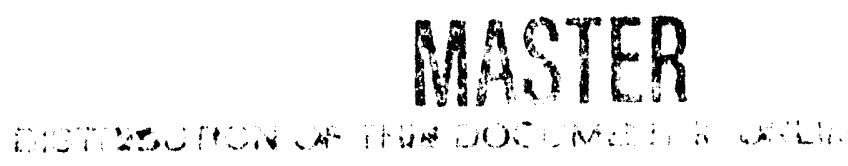




\section{Introduction}

In the United States, cancer registries have been compiling data for decades, and state vital statistics offices have been compiling birth and death data for nearly a century. Although this information has been well used for disease surveillance and various studies, it could be better exploited by making it more readily available, reducing the duplication of effort that occurs when researchers at the private, city, county, state, and federal levels work separately on their data collection and disease investigations. This report summarizes the nationwide availability of birth and death records and cancer registry data, with particular emphasis on subcounty geographic detail, such as zip code and census tract.

Birth and death data are available at the county level for the entire United States from the National Center for Health Statistics. However, county level data are inadequate for small area studies of potential environmental hazards. Hazards of current interest to the Department of Energy (DOE) include DOE facilities, nuclear power plants, and sources of electromagnetic radiation such as broadcasting towers and power lines.

For additional information about existing health data bases, see Gable $\mathrm{CB}, 1$ Flynt et al., ${ }^{2}$ and Frisch et al. ${ }^{3}$

\section{Methods}

The information compiled in this report was obtained between June and August, 1990 , by telephoning vital statistics offices and cancer registries in the United States. Special effort was made to ascertain the geographic detail and coverage of the data, with emphasis on census tract codes and zip codes. Where zip code and/or census tract data were collected, we obtained additional information regarding the completeness of the coverage and the years covered by the data. In each case, we attempted to determine the person to contact when requesting the data, and any 
special protocols required to obtain the data. In December, 1993, follow-up letters were sent to each registry and vital statistics office to verify the correctness of the data.

In Tables 1 and 2, "Data Available" refers to the earliest year for which birth and death records were collected by the state. "Data On Line" refers to the earliest year for which computer-readable birth or death records are available. "Zip Code Available" refers to the earliest year for which zip code information is available for the birth or death records. "Census Tract Available" refers to the earliest year for which census tract information is available and for which counties if not statewide.

In Table 3, "Start Date" refers to the earliest year for which cancer records were collected by the registry. "Pop'n Based?" indicates whether the registry is populationbased or when it became population-based (a registry is considered population-based if it collects cancer cases among all residents within the region covered by that registry). "Zip Code" and "Census Tract Available" serve the same purpose as in the birth and death tables.

Appendices $A, B$, and $C$ contain additional information for birth and death registries, cancer registries, and cancer registry services, respectively. "Comments" includes any additional information that pertains to data from that state. "Ordering" indicates any special procedures for obtaining data.

\section{Results}

All states have birth and death data on line except for Montana (Tables 1 and 2); three-quarters of the states have computer-readable data from 1970 forward. Zip codes are available for birth records in 44 of the 51 states, and are available for death records in 39 states (in this report, District of Columbia is considered a state). Census tracts are available for birth and death records in 22 and 20 states, respectively, but only the District of Columbia, Hawaii, Rhode Island, and Washington cover the entire geographic area. 
Table 3 indicates the availability of cancer registry data in the United States. Forty-six of the 51 states have cancer registries, and 41 of the registries (excluding Alaska) are population-based, but only 24 have data before 1980. In some states, regional population-based registries existed before state-wide reporting was in place; these are noted under "Comments" in Appendix B. In other cases, population-based reporting was instituted after the establishment of the registry. Alaska is populationbased, but the population covered is only Native Americans, Eskimos, and Aleuts. Forty-three of the cancer registries collect zip code data, but only 21 collect census tract data. Only eight states have cancer registries that collect census tract data for the entire state.

Appendices $A$ and $B$ indicate the person to contact when requesting data from that state. They also contain miscellaneous information that may be useful when ordering data.

Appendix C summarizes the cancer data services in the United States. Some of these services are shared by several states and may be helpful when planning a study involving more than one state.

\section{Discussion}

The data in the aforementioned databases can be used for case-control or cohort studies, provided that strict confidentiality requirements are met by the investigator. They are also useful for ecologic studies, where zip codes or census tracts can link birth, death, or cancer incidence data with census population data and databases of environmental exposures. For example, Jablon et al. performed an ecologic study of cancer in populations living near nuclear facilities. ${ }^{4}$ In other studies, ecologic data have been used to examine the relationship between birth defects and pesticide use by county 5 and by zip code. 6

Ecologic studies have well-known limitations. Special techniques are required 
for analyzing ecologic summary data, in order to avoid introducing bias related to the "ecologic fallacy".7 When linking an environmental exposure with the geographic distribution of a disease, it must be remembered that exposure is an average value pertaining to a geographic area and may not be generalizable to individuals within the population. Misclassification of exposure may result, reducing the likelihood of finding a significant association between exposure and disease. Other sources of misclassification bias exist; for example, zip codes for rural areas are not reliable because many people use post office boxes that may be up to 40 miles from home.

For disease codes most vital statistics offices and cancer registries use International Classification of Diseases (ICD) ${ }^{8}$ or International Classification of Diseases for Oncology (ICD-O $)^{9}$ codes. To keep pace with advances in medical knowledge, ICD and ICD-O definitions are revised approximately every ten years by international agreement. As a result, time trends of disease rates may have unavoidable discontinuities in those years when code changes occurred.

Census tract boundaries, zip code boundaries, and, to a lesser extent, county boundaries may change over time, making it more difficult to define the population at risk when using data that span a longer time period. It is important to determine that the recorded deaths and the estimated population at risk correspond to exactly the same geographic area and time period.

Racial classification is another variable that is not constant over time. The classification for whites presents a major problem: due to increasing ethnic awareness, many Hispanic Americans who identified themselves as "white" in the 1970 Census preferred to call themselves "other race" in the 1980 and 1990 Censuses. The bias affects primarily census denominators and not, for example, mortality or cancer incidence numerators. The result is an upward bias, beginning around 1980, of white disease rates in areas with large Hispanic populations. The upward bias exceeds 10 percent in some areas. ${ }^{10}$ Race-corrected population 
estimates, and not original census data, should be used as population denominators. (Race-corrected county population estimates may be purchased from Demo-Detail Inc., Richard Irwin, Director; 2303 Apple Hill Rd. Alexandria, VA 22308. (703) 7809563.)

Migration may cause spurious associations or mask real associations. Deaths are classified by location of residence at time of death, which may be ambiguous for people who have more than one residence and especially for diseases like cancer which may have long latency periods. Annual totals generally serve as numerators when calculating disease rates. In contrast, the population denominators collected by the Bureau of the Census refer to the physical location of the person on Census Day, for example April 1, 1990. In a location with seasonal population variation, for example, Nantucket County, Massachusetts, a systematic bias exists because the census population measurement is not an accurate estimate of the average annual population.11 It may be useful to understand the reciprocal case notification agreements that exist between various states and between various registries in order to recognize the impact that migration has on data from that state.

Of particular concern are effects due to the migration of military personnel. When studying an area that contains a military base, the epidemiologist must ascertain how military personnel are counted by the cancer registry, by the vital statistics office, and by the Bureau of the Census. A large military population within an area could alter the estimate of the number exposed and susceptible to disease.

It must be kept in mind that birth and death records are only a crude measure of health effects. Hexter et al. have determined that the reporting of birth defects on the birth certificate is poor. ${ }^{2}$ Furthermore, the cause of death reported on the death certificate may fail to represent the actual underlying cause of death. This discrepancy may arise when the death certificate is completed $\mathrm{L}_{j}$ the physician on duty at the time of death, and not by the patient's regular physician. For a more complete 
understanding of the disease-risk association, especially for chronic diseases, it is essential to consult the multiple cause of death section on the death certificate.

Assumptions and conclusions regarding cancer incidence data are dependent on whether or not the registry is population-based. If the registry is not populationbased, the registry population will not be representative of the area which it serves.

Although many states do not have statewide registries, useful cancer data may be obtained from individual hospital registries within that state. A list of approved hospital cancer registries is published by the Commission on Cancer of the American College of Surgeons. 13

Despite the limitations associated with ecologic analysis of routinely collected surveillance data, the insight provided by such studies is valuable. Often less expensive than other methods, ecologic studies may suggest etiologic hypotheses, and they can aid in planning more thorough investigations of the association between a suspected environmental health risk and disease.

\section{Acknowledgments}

We wish to thank Carl Quong and Robert Goldsmith for programmatic support of epidemiologic data activities in the Department of Energy. We are grateful to Harvard Holmes for project resources and personal involvement that were essential to the completion of this report. 


\section{References}

1 Gable CB. A Compendium of Public Health Data Sources. Am J Epidemiology 1990; 131(3): 381394.

2 Flynt JW, Norris CK, Zaro S, Kitchen SB, Kotler M, Ziegler A. State Surveillance of Birth Defects and Other Adverse Reproductive Outcomes. U.S. Department of Health and Human Services 1987.

3 Frisch JD, Shaw GM, Harris JA. Epidemiologic Research Using Existing Databases of Environmental Measures. Archives of Environmental Health 1990; 45(5): 303-307.

4 Jablon S, Hrubec Z, Boice J, Stone BJ. Cancer in Populations Living Near Nuclear Facilities. U.S. Department of Health and Human Services 1990.

5 Schwartz DA, LoGerfo JP. Congenital limb reduction defects in the agricultural setting. Am J Public Health 1988; 78: 654-59.

6 Grether JK, Harris JA, Neutra R, Kizer KW. Exposure to aerial Malathion application and the occurrence of congenital anomalies and low birthweight. Am J Public Health 1987; 77: 1009-10.

7 Selvin S. Two Issues Concerning the Analysis of Grouped Data. European J Epidemiology 1987; 3(3): 284-87.

8 Manual of the International Statistical Classification of Disease, Injuries, and Causes of Death, Ninth Revision. World Health Organization 1977.

9 International Classification of Diseases for Oncology, First Edition. World Health Organization 1976.

10 Merrill DW. The Socio-Economic-Environmental Demographic Information System, On-line technical documentation. Lawrence Berkeley Laboratory 1985.

11 Selvin S, Sacks ST, Merrill DW. Patterns of United States Mortality for Ten Selected Causes of Death. Lawrence Berkeley Laboratory Report LBL-10627, 1980.

12 Hexter AC, Harris JA, Roeper P, Croen LA, Krueger P, Gant D. Evaluation of the Hospital Discharge Diagnoses Index and the Birth Certificate as Sources of Information on Birth Defects. Public Health Reports 1990; 105(3): 296-306.

13 Commission on Cancer. Cancer Programs Approved 1990. American College of Surgeons, 55 East Erie Street, Chicago IL 60611. 


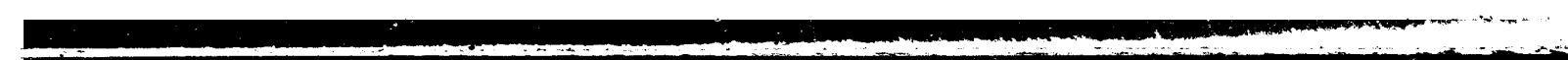


Table 1: Birth Data Availability

\begin{tabular}{|c|c|c|c|c|}
\hline State & $\begin{array}{c}\text { Data } \\
\text { Available }\end{array}$ & $\begin{array}{c}\text { Data } \\
\text { On Line }\end{array}$ & $\begin{array}{l}\text { Zip Code } \\
\text { Available }\end{array}$ & $\begin{array}{l}\text { Census Tract } \\
\text { Available }\end{array}$ \\
\hline Alabama & 1908 & 1960 & 1988 & Not available \\
\hline Alaska & 1913 & 1974 & 1968 & Not available \\
\hline Arizona & 1900 & 1950 & 1968 & 1960-Maricopa, Pima \\
\hline Arkansas & 1913 & 1985 & 1985 & Not available \\
\hline California & 1905 & 1960 & 1982 & Not available \\
\hline Colorado & 1900 & 1975 & 1975 & $\begin{array}{l}\text { 1975-Adams, Arapahoe, } \\
\text { Douglas, Denver; 1978- } \\
\text { Boulder, Jefferson, Pueblo; } \\
\text { 1987-Gilpin, Clear Creek }\end{array}$ \\
\hline Connecticut & 1897 & 1959 & 1988 & $\begin{array}{l}1981 \text {-urban areas }(85-95 \% \text { of } \\
\text { state) }\end{array}$ \\
\hline Delaware & 1920 & 1968 & 1986 & 1986-New Castle County \\
\hline District of Columbia & 1890 & 1968 & 1980 & 1968-entire district \\
\hline Florida & 1917 & 1970 & 1970 & $\begin{array}{l}\text { 1975-Broward; 1991- } \\
\text { Hillsborough }\end{array}$ \\
\hline Georgia & 1919 & $1983-92$ & 1985 & Fulton County locally entered \\
\hline Hawaii & 1853 & 1985 & N/A & 1968-entire state \\
\hline Idaho & 1911 & 1954 & 1989 & Not available \\
\hline Illinois & 1916 & 1916 & 1989 & Chicago only \\
\hline Indiana & 1907 & 1970 & 1979 & Not available \\
\hline lowa & 1880 & 1965 & 1989 & Not available \\
\hline Kansas & 1911 & 1911 & 1978 & Not available \\
\hline Kentucky & 1911 & 1976 & 1976 & Not available \\
\hline Louisiana & 1912 & 1915 & 1983 & Not available \\
\hline Maine & 1892 & 1976 & 1984 & Not available \\
\hline Maryland & 1903 & 1970 & 1986 & 1983-urban areas \\
\hline Massachusetts & 1841 & 1969 & 1986 & $\begin{array}{l}\text { 1972-Boston city; } 1986 \text {-urban } \\
\text { areas ( } 85 \% \text { of state) }\end{array}$ \\
\hline Michigan & 1867 & 1969 & 1978 & $\begin{array}{l}\text { 1970-88: Kent, Grand Rapids } \\
\text { city, Detroit city ; 1989-present, } \\
\text { not available }\end{array}$ \\
\hline
\end{tabular}


Table 1: Birth Data Availability

\begin{tabular}{|c|c|c|c|c|}
\hline State & $\begin{array}{c}\text { Data } \\
\text { Available }\end{array}$ & $\begin{array}{c}\text { Data } \\
\text { On Line }\end{array}$ & $\begin{array}{l}\text { Zip Code } \\
\text { Available }\end{array}$ & $\begin{array}{l}\text { Census Tract } \\
\text { Available }\end{array}$ \\
\hline Minnesota & 1900 & 1959 & 1989 & 1970-Minneapolis \& St. Paul \\
\hline Mississippi & 1912 & 1958 & 1979 & Not available \\
\hline Missouri & 1911 & 1968 & 1972 & $\begin{array}{l}\text { 1968-St. Louis city; 1975-St. } \\
\text { Louis county }\end{array}$ \\
\hline Montana & 1907 & N/A & N/A & Not available \\
\hline Nebraska & 1905 & 1958 & N/A & $\begin{array}{l}\text { 1970-Dakota \&Washington (to } \\
\text { 1988), Sarpy (to 1990), Douglas } \\
\text { \& Lancaster (to present) }\end{array}$ \\
\hline Nevada & 1911 & 1968 & 1991 & Not available \\
\hline New Hampshire & 1940 & 1971 & 1987 & Not available \\
\hline New Jersey & 1878 & 1967 & 1989 & Not available \\
\hline New Mexico & 1919 & 1964 & 1980 & Not available \\
\hline New York* & 1900 & 1960 & 1978 & $\begin{array}{l}\text { 1969-Albany, Broome, Erie, } \\
\text { Monroe, Nassau, Niagara, } \\
\text { Onandaga, Saratoga, West- } \\
\text { chester; 1975-Suffolk; 1982- } \\
\text { Oneida; 1985-Rensselaer, } \\
\text { Schenectady; 1987-Rockland }\end{array}$ \\
\hline New York City* & 1900 & 1968 & 1983 & New York city only \\
\hline North Carolina & 1914 & 1956 & 1988 & Not available \\
\hline North Dakota & 1893 & 1978 & 1978 & Not available \\
\hline Ohio & 1908 & 1959 & N/A & 1979-urban areas \\
\hline Oklahoma & 1908 & 1975 & 1975 & Not available \\
\hline Oregon & 1903 & 1970 & 1984 & 1978-Multnomah, Portland city \\
\hline Pennsylvania & 1906 & 1960 & 1979 & Not available \\
\hline Rhode Island & 1893 & 1968 & N/A & 1972-entire state \\
\hline South Carolina & 1915 & 1970 & 1989 & $\begin{array}{l}\text { 1970-Florence, Richland, } \\
\text { Greenville, Charleston }\end{array}$ \\
\hline South Dakota & 1905 & 1960 & 1980 & Not available \\
\hline
\end{tabular}

Note: New York state and New York City birth data are available from separate agencies. 
Table 1: Birth Data Availability

\begin{tabular}{lcccl} 
State & $\begin{array}{c}\text { Data } \\
\text { Available }\end{array}$ & $\begin{array}{c}\text { Data } \\
\text { On Line }\end{array}$ & $\begin{array}{c}\text { Zip Code } \\
\text { Available }\end{array}$ & $\begin{array}{l}\text { Census Tract } \\
\text { Available }\end{array}$ \\
\hline Tennessee & 1917 & 1960 & 1989 & 1976-Hamilton, Knox, Shelby \\
Texas & 1903 & 1964 & 1977 & Not available \\
Utah & 1905 & 1970 & 1978 & Not available \\
Vermont & 1850 & 1979 & N/A & Not available \\
Virginia & 1913 & 1975 & 1975 & Not available \\
Washington & 1907 & 1968 & 1989 & 1980 -entire state \\
West Virginia & 1917 & 1968 & 1979 & Not available \\
Wisconsin & 1907 & 1959 & 1979 & Not available \\
Wyoming & 1909 & 1978 & N/A & Not available
\end{tabular}


Table 2: Death Data Availability

\begin{tabular}{|c|c|c|c|c|}
\hline State & $\begin{array}{c}\text { Data } \\
\text { Available }\end{array}$ & $\begin{array}{c}\text { Data } \\
\text { On Line }\end{array}$ & $\begin{array}{l}\text { Zip Code } \\
\text { Available }\end{array}$ & $\begin{array}{l}\text { Census Tract } \\
\text { Available }\end{array}$ \\
\hline Alabama & 1908 & 1960 & 1988 & Not available \\
\hline Alaska & 1913 & 1977 & 1968 & Not available \\
\hline Arizona & 1900 & 1992 & 1968 & 1960-Maricopa, Pima \\
\hline Arkansas & 1913 & 1980 & 1985 & Not available \\
\hline California & 1905 & 1960 & 1989 & Not available \\
\hline Colorado & 1900 & 1975 & 1975 & $\begin{array}{l}\text { 1975-Adams, Arapahoe, } \\
\text { Douglas, Denver; 1978- } \\
\text { Boulder, Jefferson, Pueblo; } \\
\text { 1987-Gilpin, Clear Creek }\end{array}$ \\
\hline Connecticut & 1897 & 1949 & 1989 & $\begin{array}{l}1981 \text {-urban areas }(85-95 \% \text { of } \\
\text { state) }\end{array}$ \\
\hline Delaware & 1930 & 1968 & 1986 & 1986-New Castle County \\
\hline District of Columbia & 1890 & 1968 & 1980 & 1968-entire district \\
\hline Florida & 1917 & 1970 & 1991 & 1975-Broward \\
\hline Georgia & 1919 & $1983-92$ & $N / A$ & Not available \\
\hline Hawaii & 1853 & 1991 & N/A & 1968-entire state \\
\hline Idaho & 1911 & 1954 & 1989 & Not available \\
\hline Illinois & 1916 & 1916 & 1965 & Not available \\
\hline Indiana & 1900 & 1970 & 1979 & Not available \\
\hline lowa & 1880 & 1954 & N/A & Not available \\
\hline Kansas & 1911 & 1989 & 1978 & Not available \\
\hline Kentucky & 1911 & 1976 & 1987 & Not available \\
\hline Louisiana & 1912 & 1944 & 1978 & Not available \\
\hline Maine & 1892 & 1970 & N/A & Not available \\
\hline Maryland & 1903 & 1970 & 1986 & 1983-urban areas \\
\hline Massachusetts & 1841 & 1969 & 1987 & $\begin{array}{l}\text { 1972-Boston city; } 1987 \text {-urban } \\
\text { areas ( } 85 \% \text { of state) }\end{array}$ \\
\hline Michigan & 1867 & 1968 & 1989 & $\begin{array}{l}\text { 1970-Kent, Grand Rapids city, } \\
\text { Detroit city }\end{array}$ \\
\hline
\end{tabular}


Table 2: Death Data Availability

\begin{tabular}{|c|c|c|c|c|}
\hline State & $\begin{array}{c}\text { Data } \\
\text { Available } \\
\end{array}$ & $\begin{array}{c}\text { Data } \\
\text { On Line } \\
\end{array}$ & $\begin{array}{l}\text { Zip Code } \\
\text { Available }\end{array}$ & $\begin{array}{l}\text { Census Tract } \\
\text { Available }\end{array}$ \\
\hline Minnesota & 1900 & 1944 & 1992 & 1970- Minneapolis \& St. Paul \\
\hline Mississippi & 1912 & 1958 & N/A & Not available \\
\hline Missouri & 1911 & 1968 & 1972 & 1975-St. Louis city and county \\
\hline Montana & 1907 & N/A & N/A & Not available \\
\hline Nebraska & 1905 & 1958 & N/A & $\begin{array}{l}\text { 1970-Dakota \&Washington (to } \\
\text { 1988), Sarpy (to 1990), Douglas } \\
\text { \& Lancaster (to present) }\end{array}$ \\
\hline Nevada & 1911 & 1968 & 1991 & Not available \\
\hline New Hampshire & 1940 & 1968 & N/A & Not available \\
\hline New Jersey & 1878 & 1967 & 1989 & Not available \\
\hline New Mexico & 1919 & 1962 & 1989 & Not available \\
\hline New York* & 1900 & 1960 & 1978 & $\begin{array}{l}\text { 1969-Albany, Broome, Erie, } \\
\text { Monroe, Nassau, Niagara. } \\
\text { Onandaga, Saratoga, West- } \\
\text { chester; 1975-Suffolk; 1982- } \\
\text { Oneida; 1985-Rensselaer, } \\
\text { Schenectady; 1987-Rockland }\end{array}$ \\
\hline New York City ${ }^{\star}$ & 1900 & 1968 & 1983 & 1983-New York City only \\
\hline North Carolina & 1914 & 1956 & 1988 & Not available \\
\hline North Dakota & 1893 & 1978 & 1978 & Not available \\
\hline Ohio & 1908 & 1959 & N/A & 1979-urban areas \\
\hline Oklahoma & 1908 & 1975 & 1975 & Not available \\
\hline Oregon & 1903 & 1970 & 1986 & 1978-Multnomah, Portland city \\
\hline Pennsylvania & 1906 & 1960 & 1979 & Not available \\
\hline Rhode Island & 1943 & 1968 & N/A & 1972-entire state \\
\hline South Carolina & 1915 & 1970 & 1989 & $\begin{array}{l}\text { 1970-Florence, Richland, } \\
\text { Greeneville, Charleston }\end{array}$ \\
\hline South Dakota & 1905 & 1960 & 1988 & Not available \\
\hline Tennessee & 1917 & 1949 & 1989 & 1976-Hamilton, Knox, Shelby \\
\hline
\end{tabular}

Note: New York state and New York City death data are available from separate agencies. 


\section{Table 2: Death Data Availability}

\begin{tabular}{lcccl} 
State & $\begin{array}{c}\text { Data } \\
\text { Available }\end{array}$ & $\begin{array}{c}\text { Data } \\
\text { On Line }\end{array}$ & $\begin{array}{c}\text { Zip Code } \\
\text { Available }\end{array}$ & $\begin{array}{l}\text { Census Tract } \\
\text { Available }\end{array}$ \\
\hline Texas & 1903 & 1964 & 1989 & Not available \\
Utah & 1905 & 1956 & 1978 & Not available \\
Vermont & 1850 & 1968 & N/A & Not available \\
Virginia & 1913 & 1975 & 1975 & Not available \\
Washington & 1907 & 1968 & 1988 & 1980 -entire state \\
West Virginia & 1917 & 1948 & 1979 & Not available \\
Wisconsin & 1907 & 1958 & 1989 & Not available \\
Wyoming & 1909 & 1978 & N/A & Not available
\end{tabular}




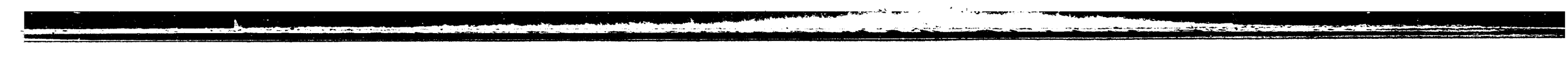


Table 3: Cancer Data Availability

\begin{tabular}{|c|c|c|c|c|c|}
\hline State & Agency & $\begin{array}{l}\text { Start } \\
\text { Date }\end{array}$ & $\begin{array}{l}\text { Pop'n } \\
\text { Based? }\end{array}$ & $\begin{array}{l}\text { Zip } \\
\text { Code }\end{array}$ & $\begin{array}{l}\text { Census Tract } \\
\text { Available }\end{array}$ \\
\hline Alabama & Alabama Dept. of Health & N/A & N/A & N/A & N/A \\
\hline Alaska & Alaska Area Native Health Service & 1969 & Yes & N/A & Not available \\
\hline Arizona & Arizona Cancer Registry & 1980 & 1992 & 1980 & $\begin{array}{l}\text { Available for childhood \& testicular } \\
\text { cancer cases diagnosed } 1965 \text { to } 1989 \text { in } \\
\text { Maricopa \& Pima counties }\end{array}$ \\
\hline Arkansas & Central Arkansas Radiation Therapy Institute & 1982 & No & N/A & Not available \\
\hline California & California Cancer Surveillance Section & 1973 & Yes & 1973 & $\begin{array}{l}\text { 1973-San Francisco Bay Area \& Los } \\
\text { Angeles; 1984-Orange County; } 1988 \text { - } \\
\text { statewide }\end{array}$ \\
\hline Colorado & Colorado Central Cancer Registry & 1968 & 1988 & 1968 & $\begin{array}{l}\text { 1979-Adams, Arapahoe, Boulder, } \\
\text { Denver, Jefferson, Douglas, \& Gilpin } \\
\text { counties }\end{array}$ \\
\hline Connecticut & Connecticut Tumor Registry & 1935 & Yes & 1935 & $\begin{array}{l}\text { 1973-83: partially available; } 1984-88: \\
\text { entire state ( } 80 \% \text { complete) }\end{array}$ \\
\hline Delaware & Delaware State Tumor Registry & 1972 & Yes & 1972 & 1980-entire state \\
\hline District of Columbia & Central Cancer Registry & 1950 & Yes & 1950 & Available for entire district \\
\hline Florida & Cancer Epidemiology, HSDE & 1979 & 1981 & 1979 & $\begin{array}{l}\text { 1981-available for } 85 \% \text { of state; } \\
\text { unavailable for rural routes \& P.O. boxes }\end{array}$ \\
\hline Georgia & Georgia Center for Cancer Statistics & 1969 & No & 1975 & $\begin{array}{l}\text { 1975-Fulton, Dekalb, Clayton, Gwinnett, } \\
\text { \& Cobb counties }\end{array}$ \\
\hline Hawaii & Hawaii Tumor Registry & 1960 & 1960 & 1960 & 1979-entire state \\
\hline Idaho & Cancer Data Registry of Idaho & 1969 & Yes & 1969 & Not available \\
\hline Illinois & Illinois Cancer Registry & 1985 & Yes & 1985 & 1985-entire state except for rural routes \\
\hline Indiana & State Cancer Registry & 1987 & 1987 & 1987 & Not available \\
\hline lowa & State Health Registry of lowa & 1973 & Yes & 1973 & $\begin{array}{l}\text { 1978-Dubuque, Woodbury, Black Hawk, } \\
\text { Linn, Scott, Polk, Pottawattamie, \& parts } \\
\text { of Warren and Dallas counties }\end{array}$ \\
\hline Kansas & Cancer Data Service & 1969 & 1983 & 1970 & Not available \\
\hline Kentucky & Kentucky Cancer Registry & 1986 & 1991 & 1986 & Not available \\
\hline Louisiana & Louisiana Tumor Registry & 1974 & 1974 & 1974 & $\begin{array}{l}\text { 1974-Orleans, Jefferson, \& St. Bernard } \\
\text { parishes; } 1984 \text {-southern state; } 1988- \\
\text { entire state }\end{array}$ \\
\hline Maine & Maine Cancer Registry & 1983 & Yes & 1983 & Not available \\
\hline
\end{tabular}


Table 3: Cancer Data Availability

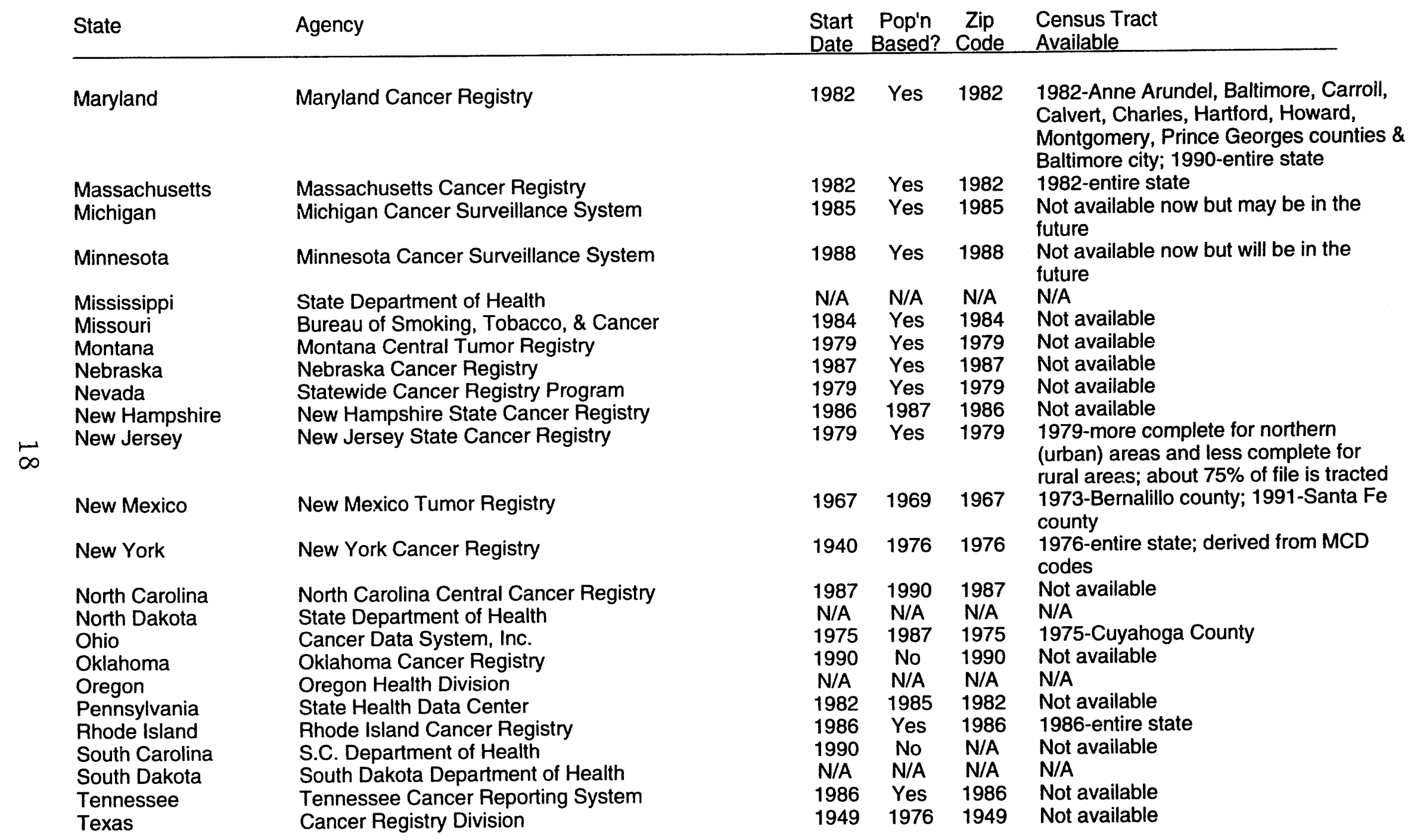


Table 3: Cancer Data Availability

\begin{tabular}{|c|c|c|c|c|c|}
\hline State & Agency & $\begin{array}{l}\text { Start } \\
\text { Date }\end{array}$ & $\begin{array}{l}\text { Pop'n } \\
\text { Based? }\end{array}$ & $\begin{array}{l}\text { Zip } \\
\text { Code }\end{array}$ & $\begin{array}{l}\text { Census Tract } \\
\text { Code }\end{array}$ \\
\hline Utah & Utah Cancer Registry & 1966 & 1966 & 1966 & $\begin{array}{l}\text { 1973-Utah, Salt Lake, Davis, \& Weber } \\
\text { counties; 1978-Toole County }\end{array}$ \\
\hline Vermont & Vermont Cancer Registry & 1993 & 1993 & 1993 & Not available \\
\hline Virginia & Virginia Tumor Registry & 1970 & 1990 & 1970 & Not available \\
\hline Washington & Cancer Surveillance System & 1991 & Yes & 1974 & $\begin{array}{l}\text { 1974-King, Pierce, Snohomish, } \\
\text { Thurston, Whatcom, \& Kitsap counties }\end{array}$ \\
\hline West Virginia & Office of Epidemiology & 1991 & Yes & 1991 & Not available. \\
\hline Wisconsin & Wisconsin Cancer Reporting System & 1976 & 1978 & 1976 & Not available \\
\hline Wyoming & Wyoming Central Tumor Registry & 1962 & 1962 & 1962 & Not available \\
\hline
\end{tabular}




\section{Appendix A: Birth \& Death Data Ordering Information}

\author{
Alabama \\ Dorothy Harshbarger, State Registrar \\ Center for Health Statistics \\ Alabama State Health Department \\ P.O. Box 5625 \\ Montgomery AL 36103-5625 \\ (205) $013-5426$
}

\section{Alaska}

Al Zangri, Chief

Bureau of Vital Statistics

Alaska Department of Health

P.O. Box H

Juneau AK 99811-0675

(907) 465-3992

\section{Arizona}

Mary Symcox

Office of Vital Records

Arizona State Department of Health

P.O. Box 3887

Phoenix AZ 85030

(602) 542-1084

\section{Comments:}

Alabama application for Release of Vital Records and signed confidentiality statement must be submitted and approved before records with individual identifiers are released.

\section{Ordering:}

Comments:

Send request with research summary \& protocol.

Ordering:

If approved, cost is $\$ 70 / \mathrm{hr}$ for on-line data pulls.

Off-line priced separately depending on request scope.

\section{Comments:}

Approval by Dept's Subject Review Committee required. Signed confidentiality statement required. No follow-back permitted. Identifiers on records must not be used or contacted.

\section{Ordering:}

Non-certified copies \$2 each. When submitting protocol, include how you maintain confidentiality of records \& what final disposition will be.

Comments:

Arkansas

Werner Haney, Director of Vital Statistics Center for Health Statistics, Slot 19

Arkansas Department of Health

4815 West Markham

Little Rock AR 72205-3867

(501) $661-2510$

\section{California}

Charles Chan

Vital Statistics Section

Department of Health Services

714 P Street, Room 1494

Sacramento CA 95814

(916) 657-3057

Fax: (916) 324-5599
Comments:

Ordering:

Cost: $\$ 175$ for first year, $\$ 50$ for each additional year. 


\section{Colorado}

Carol Garrett, Section Chief

Office of Vital Statistics

Colorado Department of Health

4300 Cherry Creek Drive South

HSUR - HS - A5

Denver CO 80222-1530

(303) $692-2160$

Fax: (303) 782-0095

\section{Connecticut}

Kathryn E. Friedrich

Registrar of Vital Records

Department of Public Health \& Addiction Services

150 Washington Strost

Hartford CT 06106

(203) 566-6545

\section{Delaware}

Mary E. Cannon

Health Planning and Resources Management

Department of Health and Social Services

P.O. Box 637

Dover DE 19903

(302) $736-4776$

\section{District of Columbia}

Carl W. Wilson, Chief

Research and Statistics Division

D.C. Department of Human Resources

613 G Street, 9th Floor

Washington DC 20001

(202) 727-0682

Fax: (202) 727-3396

\section{Florida}

Kenneth T. Jones, Acting Chief

Office of Vital Statistics

P.O. Box 210

Jacksonville FL 32231-0042

(904) 359-6929

Georgia

Michael R. Lavoie, Director

Vital Records Service

Department of Human Resources

Room $217 \mathrm{H}$

Atlanta GA 30334

(404) $656-4750$
Comments:

Ordering:

Comments:

Birth \& death data requests for research must be reviewed \& approved by agency's Human Investigations Committee.

Ordering:

Call for copy of HIC protocol.

\section{Comments:}

All data requests must be reviewed and may require Board of Health approval. Confidentiality assurances are required.

Ordering:

Comments:

Ordering:

Comments:

Requests involving confidential information require study protocol and Data Purchase and Use

Agreement to be submitted for review by the State.

Ordering:

Special programming requests are on a fee-forservices basis.

Comments:

Hold-harmless agreement required. If contact with subjects is necessary, IRB or DHR Human Subjects Committee approval needed.

Ordering:

Send a written summary with the protocol. 
Hawaii

Dr. Alvin Onaka, State Registrar

Office of Health Status Monitoring

Hawaii Department of Health

P.O. Box 3378

Honolulu HI 96801

(808) 548-6454

Idaho

Janet M. Wick, Head Statistician

Bureau of Vital Statistics

Cooperative Center for Health Statistics

Statehouse

Boise ID 83720

(208) 334-5978

Illinois

Larry Davis

Division of Vital Records

State Department of Public Health

605 West Jefferson St.

Springfield IL 62702-5097

(217) $782-6553$

Indiana

James M. Miklos, Administrative Officer

Planning \& Information Services

Indiana State Departmentof Health

1330 West Michigan Street

PO Box 1964

Indianapolis IN 46206-1964

(317) 633-8515

lowa

Larry Coghlan

lowa Department of Health

Lucas State Office Building

Des Moines IA 50319

(515) $281-3583$

\section{Kansas}

Dr. Lorne Phillips, State Registrar

Center for Health \& Environmental Statistics

Kansas Health Department

Landon State Offiice Building

900 SW Jackson St.

Topeka KS 66612

(913) 296-8075
Comments:

Dept requests a gratis copy of any report using Hawaii data.

Ordering:

Request copy of confidentiality form before ordering. Birth data requests must be reviewed by Data Committee.

Comments:

Data available on tape from 1954. Limited

disclosure of confidential information.

Ordering:

Research application required.

Comments:

Ordering:

Comments:

Before 1979, zip code data are incomplete.

Ordering:

Cost: approximately $\$ 600 /$ yr. Send study protocol with request.

Comments:

Birth data are available between 1959-61 \& since 1965.

Ordering:

Research agreement required. Fee for copy or search is $\$ 10.00$.

Comments:

Zip code reporting for deaths is optional \& was optional for births prior to 1989.

Ordering:

Fee must be paid prior to processing of request. 
Kentucky

Mrs. Jo Warren

Health Data Branch

Division of Vital Records \& Health Development 275 East Main Street, CHR Building

Frankfort KY 40621

(502) $564-2757$

\section{Louisiana}

Mr. William Barlow, State Registrar

Office of Vital Statistics

Louisiana Department of Health

P.O. Box 60630

New Orleans LA 70160

(504) 568-8353

Maine

Ellen Naor, State Registrar

Office of Data, Research, and Vital Statistics

35 Anthony Avenue

Statehouse Station \#11

Augusta ME 04333-0011

(207) 624-5445

\section{Maryland}

Ms. Julia Davidson-Randall, State Registrar Department of Health and Mental Hygiene

General Services Administration

201 West Preston Street, Room 519

Baltimore MD 21201

(410) 225-5830

Fax: (410) 333-5958

Massachusetts

Elaine Trudeau, State Registrar

Registry of Vital Records \& Statistics

150 Tremont Street, Room B-3

Boston MA 02111

(617) 727-0036

Fax: (617) 727-8108

Michigen

George Van Amburg

Office of the State Registrar

Michigan Department of Public Health

P.O. Box 30195

( ansing MI 48909

(517) $335-8675$
Comments:

Ordering:

Comments:

Death records are transferred to the Louisiana

State Archives after 50 years. Research regulations control disclosure of birth \& death data which include identifiers.

Ordering:

Comments:

Ordering:

Comments:

Ordering:

Comments:

Ordering:

Approval required for birth data \& suggested for death data.

Comments:

Ordering:

Researchers must submit study protocol to receive research fee rate. 
Minnesota

John Oswald

Office of Health Statistics

State Department of Health

717 Delaware Street SE

Minneapolis MN 55440

(612) 623-5187

Fax: (612) 623-5043

\section{Mississippi}

Nita Cox Gunter

Bureau of Information Resources

Mississippi State Department of Health

P.O. Box 1700

Jackson MS 39215-1700

(601) $960-7977$

\section{Missouri}

Wayne Schramm, Chief

Bureau of Health Data Analysis

Missouri Department of Health

P.O. Box 570

Jefferson City MO 65102

(314) $751-6278$

Fax: (314) 526-4102

\section{Montana}

Sam Sperry, Chief

Vital Statistics Bureau

Montana Department of Health

PO Box 200901

Helena MT 59620

(406) 444-4228

\section{Nebraska}

Mark Miller

Division of Health Data Systems

Nebraska State Health Department

301 Centennial Mall South

P.O. Box 95007

Lincoln NE 68509-5007

(402) 471-0355

\section{Nevada}

Mary Howard

Office of Vital Statistics

Nevada State Department of Health

505 East King Street

Carson City NV 89710

(702) $687-4480$
Comments:

Ordering:

There is a charge for computerized files.

Tomments:

Research \& epidemiology studies require NDI approval.

Ordering:

Comments:

Ordering:

Comments:

Ordering:

Comments:

Ordering:

Must detail the data fields requested and the purpose of acquiring data and how or in what form they will be published. If request meets proper purpose laws, a lenal agreement must be signed.

\section{Comments:}

Ordering: 
New Hampshire

Mr. Frank Novak, Acting State Registrar Bureau of Vital Records and Health Statistics Health \& Human Services Building

6 Hazen Drive

Concord NH 03301-6527

(603) 271-4651

Fax: (603) 271-3447

New Jersey

Charles Karkut, State Registrar

Bureau of Vital Statistics

C.N. 370

Trenton NJ 08625

(609) 292-8085

Fax: (609) 392-4929

New Mexico

Betty Hileman, Chief

Bureau of Vital Statistics

New Mexico Health \& Environment Dept.

1190 St. Frances Drive

Santa Fe NM 87503

(505) 827-2342

New York (excl New York City)

Gene Therriault, Director

Bureau of Biometrics

New York Department of Health

Empire State Plaza Concourse

Room C-144

Albany NY 12337-0044

(518) 474-3189

Fax: (518) 486-1630

New York City

Dr. Stephen Stellman, Asst Commissioner Biostatistics \& Epidemiological Research

New York City Department of Health

125 Worth Street

New York City NY 10013

(212) $566-6371$

North Carolina

Kathryn McLawhorn

Center for Health \& Environmental Statistics

P.O. Box 27687

Cottor Building

Raleigh NC 27611-7687

(919) $733-4728$
Comments:

Ordering:

Comments:

Ordering:

Comments:

Severe confidentiality \& follow-back restrictions.

NDI required for death data. Other uses by proposal approval only.

Ordering:

Lengthy process.

Comments:

For New York city, see separate record.

Ordering:

Confidentiality statement is required.

Comments:

For NY state outside of NY city, see separate record.

Ordering:

Comments:

Data prior to 1968 are not readily available.

Ordering: 


\section{North Dakota}

David Mayer

Division of Vital Statistics

North Dakota Dept Health \& Consolidated Labs

State Capitol Building

Judicial Wing, 600 East Blvd.

Bismarck ND 58505-0200

(701) 224-2314

Fax: (701) 224-4727

Ohio

Mary F. Smith, Statistical Analysis Unit

Ohio Department of Health

Health Policy Data Center

P.O. Box 118

Columbus $\mathrm{OH} 43266-0118$

(614) 644-7808

\section{Oklahoma}

Roger Pirrong, State Registrar

Bureau of Vital Statistics

Vital Records Division

Department of Health

P.O. Box 53551

Oklahoma City OK 73152

(405) 271-4040

\section{Oregon}

Edward Johnson II, State Registrar

Center for Health Statistics

P.O. Box 14050

Portland OR 97214-0050

(503) $731-4109$

\section{Pennsylvania}

Patricia W. Potrzebowski, Director

Division of Health Statistics and Research

State Health Data Center

Pennsylvania Department of Health

Harrisburg PA 17108

(717) $783-2548$

Rhode Island

Roberta Chevoya

Division of Vital Records

101 Cannon Building

3 Capitol Hill

Providence RI 02908

(401) $277-2812$
Comments:

Ordering:

Comments:

Ordering:

Specify request in detail to obtain cost \& time estimate.

Comments:

Ordering:

Cost estimate requires detailed written request.

Comments:

Ordering:

Cost: $\$ 300$ per data year.

Comments:

Minor civil division codes available since 1961.

Ordering:

Comments:

Ordering:

Cannot create public use tapes. Confidentiality agreement required. Fee for specially tailored reports. 
South Carolina

\section{Betsy Barton}

Office of Vital Records \& Public Health Statistics South Carolina Dept. of Health \& Env. Control 2600 Bull Street

Columbia SC 29201

(803) 734-4681

Fax: (803) 734-5131

South Dakota

Dorris Donner, State Registrar

Vital Records

Department of Health

445 East Capitol

Pierre SD 57501-3185

(605) 773-4961

\section{Tennessee}

Jean Moss, Director

Health Statistics \& Information

Division of Information Resources

Department of Health

419 Cordell Hull Building

Nashville TN 37247-0360

(615) $741-1954$

Fax: (615) 532-1886

Texas

Mr. Tom Pollard, Director

Bureau of Vital Statistics

Texas Department of Health

1100 West 49th

Austin TX 78756

(512) $458-7362$

Utah

John E. Brockert, State Registrar

Bureau of Vital Records

Utah Department of Health

P.O. Box 16700

Salt Lake City UT 84116-0700

(801) 538-6186

\section{Vermont}

Charles Bennett, Ph.D.

Division of Public Health Analysis \& Policy

Vermont Department of Public Health

P.O. Box 70

Burlington VT 05402

(802) $863-7300$

Fax: (802) 863-7425
Comments:

Restricted by law/regulation.

Ordering:

Send written requests with specification as to the data items needed.

Comments:

Ordering:

Specify data items needed. Confidentiality required.

Comments:

Ordering:

Comments:

Birth data on line 1926-28, and since 1941. Birth

data confidential until age 50; death data confidential for 25 years after death.

Ordering:

Send fee and request for confidential information to Committee Request for Personal Data.

Comments:

Zip code not veritied.

Ordering:

Send written requests with specification as to the data items needed and the years of data required.

Comments:

City and town codes are available.

Ordering: 
Virginia

Ron Hyman

Center for Health Statistics

Virginia Department of Health

109 Governor Street, Room 307

Richmond VA 23219

(804) 786-6206

Fax: (804) $371-4800$

Washington

Craig Parker, Research Analyst

Center for Health Statistics, MS-ET-14

Department of Health

1112 Quince SE

Olympia WA 98504-7814

(206) 586-6008

Fax: (206) 753-4135

West Virginia

Chuck Bailey, Jr., State Registrar

Vital Statistics Office

West Virginia Department of Health

Building 3, Capitol Complex

Charleston WV 25305

(304) 558-0155

Fax: (304) 558-1051

\section{Wisconsin}

Clare Schneider

Center for Health Statistics

P.O. Box 309

Madison WI 53701

(608) 267-7808

Fax: (608) 267-0352

Wyoming

Cindy McCaffrey

Vital Records

Wyoming Department of Health

Hathaway Building

Cheyenne WY 82002

(307) $777-7591$
Comments:

Ordering:

Comments:

Ordering:

Comments:

Ordering:

Research agreement required. Variable costs.

Death tape $\$ 100$ plus $\$ 10$ for each year included.

Comments:

Ordering:

Fee for data on tape is approximately 2.5 cents/record, plus computer costs.

Comments:

Ordering: 


\section{Appendix B: Cancer Registry Data Ordering Information}

\section{Alabama}

Charles H. Woernle, MD

Bureau of Disease Control

Alabama Department of Public Health

434 Monroe Street \#900

Montgomery AL 31630-3017

(205) 613-5325

Fax: (205) 288-5021

Alaska

Dr. Anne Lanier

Office of Community Health Services

Alaska Area Native Health Service

250 Gambell Street

Anchorage AK 99501

(907) 271-4011

\section{Arizona}

Georgia Armenta Yee, Manager

Arizona Cancer Registry

Department of Health Services

$1400 \mathrm{~W}$. Washington St

Phoenix AZ 85007

(602) $542-7320$

Fax (602) 542-1753

\section{Arkansas}

Dr. Deborah O. Erwin

Central Arkansas Radiation Therapy Institute (CARTI)

P.O. Box 5210

Little Rock AR 72215

(501) 644-8573

\section{California}

John L. Young, Jr., Dr.P.H., Chief

California Cancer Surveillance System

Department of Health Services

PO Box 942732

Sacramento CA 94234-7320

(916) 327-4663:

Fax: (916) 327-4657

\section{Colorado}

Mrs. Robin Bott, Director

Colorado Central Cancer Registry

State Department of Health

4300 Cherry Creek Drive South

Denver CO 80222

(303) 692-2542

Fax: (303) 782-0095
Comments:

Registry data exist on microfiln only for 1960's and 1970 's. There are currently 22 hospital registries in the state.

Ordering:

Comments:

State does not have a registry; cancer cases collected for Native Americans (Indians, Eskimos, Aleuts) only since 1969.

Ordering:

Comments:

Estimated completeness for 1990 is $76 \%$.

Mandatory reporting began 1992. Uses Rocky

Mountain Cancer Data System.

Ordering:

Comments:

Estimated 75\% completeness. There are 13 hospital registries in the state.

Ordering:

Comments:

San Francisco area has been part of SEER since 1973. Statewide reporting since1988. Has reciprocal reporting agreement with Nevada.

Ordering

Comments:

Denver area population-based since 1979. Statewide repor.ing since 1988.

Ordering: 
Connecticut

Mr. John T. Flannery, Director

Connecticut Tumor Registry

Dept. of Public Health \& Addiction Services

150 Washington Street

Hartford CT 06106

(203) 566-3975

\section{Delaware}

Carol Marker, Program Administrator

Delaware State Tumor Registry

3000 Newport Gap Pike

Wilmington DE 19808

(302) 995-8606

Fax: (302) 995-8689

District of Columbia

Director

Cancer Control Bureau

425 I Street, N.W., Room 2001

Washington DC 20001

(202) $576-8888$

\section{Florida}

Mr. Douglas G. Palin

Cancer Epidemiology, HSDE

Dept. of Health \& Rehabilitative Services

State Health Office, 1317 Winewood Boulevard

Tallahassee FL 32399-0700

(904) 488-2907

Fax: (904) 922-9299

Georgia

Dr. Jonathon M. Liff, Deputy Director

Georgia Center for Cancer Statistics

Dept. of Epidemiology \& Biostatistics

Emory University School of Public Health

1462 Clifton Road, N.E.

Atlanta GA 30329

(404) 727-8713

Fax: (404) 727-8737

\section{Hawaii}

Dr. Marc Goodman

Hawaii Tumor Registry

1236 Lauhala Street, Suite 402

Honolulu HI 96813

(808) $586-9750$

Fax: (808) 587-0024

Idaho

Stacey Peterson, ART, CTR, Director

Cancer Data Registry of Idaho

802 West Bannock, Suite 500

Boise ID 83702-5842

(208) $338-5100$

Fax: (208) 338-7800
Comments:

Joined SEER Program in 1973. ZIP code not available on computer before 1992, but available on paper abstracts.

Ordering:

Comments:

Uses Rocky Mountain Cancer Data System.

Ordering:

Comments:

Ordering:

Comments:

Ordering:

\section{Comments:}

Metropolitan Atlanta has been part of SEER since 1975 , rural environs since 1978 . Voluntary reporting since 70 's is about $50 \%$ complete. Data since 1980 are on line.

Ordering:

Comments:

Joined SEER Program in 1973.

Ordering:

Comments:

Includes Idaho residents treated out-of-state \& non-residents treated in Idaho. Uses Rocky Mountain Cancer Data System.

Ordering: 


\section{Illinois}

Holly L. Howe, Ph.D., Chief

Division of Epidemiologic Studies

Illinois Department of Public Health 605 West Jefferson Street

Springfield IL 62761

(217) $785-1873$

Fax: (217) 524-1770

Indiana

Martha Graves, RRA, CTR

State Cancer Registry Indiana State Board of Heaith

P.O. Box 1964

1330 West Michigan Street

Indianapolis IN 46206-1964

(317) 633-8424

Fax: (317) 633-0847

lowa

Mrs. Kathleen McKeen, Admin. Dir. State Health Registry of lowa 100 Westlawn Bldg. S.

lowa City IA 52242

(319) 335-8609

Fax: (319) $335-8610$

\section{Kansas}

Dr. Frederick F. Holmes, Director

Cancer Data Service

University of Kansas Medical Center

College of Health Science

3901 Rainbow Boulevard

Kansas City KS 66160-7312

(913) 588-4725

Fax: (913) 588-4701

Kentucky

Mr. Thomas C. Tucker

Kentucky Cancer Registry

Lucille Parker Marky Cancer Center

800 Rose St.

Lexington KY 40536-0093

(606) 257-4582

Fax: (606) 258-1902

\section{Louisiana}

Dr. Vivien W. Chen

Louisiana Tumor Registry

Louisiana Department of Health

Room 305, P.O. Box 60630

New Orleans LA 70160-0630

(504) 568-2616

Fax: (504) 568-8650
Comments:

Ordering:

Requests must be submitted in writing. Confidential data requests must follow ISCR guidelines.

Comments:

Some data exist before 1987, but are incomplete.

Ordering:

Comments:

Joined SEER Program in 1973. Some cancer data collected 1969-71.

Ordering:

Comments:

Zip code data not reliable in rural areas because of the use of PO boxes.

Ordering:

Comments:

Ordering:

Comments:

Orleans, Jefferson, \& St. Bernard parish data available since 1974 , southern state since 1983 , statewide data since 1988.

Ordering: 
Maine

Ms. E. Melanie Lanctot

Maine Cancer Registry

157 Capitol St.

State House, Station \#11

Augusta ME 04333

(207) 287-5187

Fax: (207) 287-4172

\section{Maryland}

Dorothy Damron

Maryland Cancer Registry

University of Maryland at Baltimore

Dept. of Epidemiology \& Preventive Medicine

660 W. Redwood St., 324 Howard Hall

Baltimore MD 21201

(410) 706-6447

Fax: (410) 706-8013

\section{Massachusetts}

Susan Gershman, Director

Massachusetts Cancer Registry

Department of Public Health

150 Tremont Street, 5th Floor

Boston MA 02111-1126

(617) $727-9291$

\section{Michigan}

George Van Amburg

Michigan Cancer Surveillance System

P.O. Box 30195, 3500 North Logan

Lansing MI 48909

(517) $335-8677$

\section{Minnesota}

Sally Bushhouse, DVM, MPH

Minnesota Cancer Surveillance System

Minnesota Department of Health

717 Delaware SE

Minneapolis MN 55440

(612) 623-5216

Fax: (612) 623-5743

Mississippi

Cindy Widdig

Cancer Consultant

State Department of Health

PO Box 1700

2423 N. State Street

Jackson MS 39205

(601) $960-7854$
Comments:

Ordering:

If identifiers are needed, contact registry for copy of protocol.

Comments:

Ordering:

Comments:

Ordering:

Comments:

Ordering:

Dependent upon the nature of the data request, may require submission of a protocol $\&$ an application for review by an independent scientific advisory panel.

\section{Comments:}

Ordering:

Call to discuss the request prior to sending anything written.

\section{Comments:}

There are 11 hospital registries in the state. 
Missouri

Dr. Jian C. Chang, PhD.

Bureau of Smoking, Tobacco, \& Cancer

Missouri Department of Health

201 Business Loop 70 West

Columbia MO 65203

(314) 876-3224

Fax: (314) 443-3592

\section{Montana}

Debbi Hellhake

Montana Central Tumor Registry

Dept. of Health \& Environmental Sciences

Cogswell Building C118

Helena MT 59620

(406) 444-2.618

Fax: (406) 444-1803

\section{Nebraska}

Monica Seeland, RRA, Health Data Manager Nebraska Cancer Registry

Department of Health

Omaha NE 68509-5007

(402) 390-0352

Nevada

Phyllis Wiggins, Director

Nevada Statewide Cancer Registry Program

620 Belrose St.

Las Vegas NV 89158

(702) 486-5065

New Hampshire

Dr. Marguerite Stevens

New Hampshire Cancer Registry

PO Box 186

Hanover NH 03775-3802

(603) 650-1106

Fax: (603) 650-1493

New Jersey

Debra M. Harlan, Director

Epidemiology \& Research Services

Cancer Registry Program

New Jersey Department of Health

3635 Quaker Bridge Road, CN 369

Trenton NJ 08625-0369

(609) 588-3500

Fax: (609) 588-7431
Comments:

Ordering:

Comments:

Ordering:

Send copy of the study protocol with request for data.

Comments:

Regulations for release of information for research purposes are in effect.

Ordering:

Comments:

Uses Rocky Mountain Cancer Data System. Has reciprocal notification agreement with California.

Ordering:

Comments:

Ordering:

Comments:

Four-county SEER data available 1984-88; statewide data since 1979.

Ordering:

To obtain data, call Betsy Kohler, then send request. 
New Mexico

Dr. Charles R. Key, Med. Director

New Mexico Tumor Registry

900 Camino de Salud, N.E.

Albuquerque NM 87131

(505) 277-5541

Fax: (505) $277-8572$

\section{New York}

Mark S. Baptiste, Ph.D.

New York Cancer Registry

Department of Health, Office of Public Health

Corning Tower, Room 536, Empire State Plaza

Albany NY 12237-0683

(518) 474-2255

Fax: (518) 474-2086

\section{North Carolina}

Dr. Tim Aldrich or Dale Herman

North Carolina Central Cancer Registry

Center for Health \& Environmental Statistics

Dept of Environment, Health \& Natural Resources

P.O. Box 29538

Raleigh NC 27626-0538

(919) $715-4555$

\section{North Dakota}

Barbara Burgum Lee, Coordinator

Cancer Prevention \& Control Program

ND State Dept. of Health \& Consolidated Labs

Division of Health Promotion \& Education

600 E. Boulevard Avenue

Bismarck ND 58505-0200

(701) 224-2333

Ohio

Felicia Cammarata, Vice President

Cancer Data System, Inc.

1226 Huron Road

Cleveland $\mathrm{OH} 44115$

(216) 696-6900

Fax: (216) $781-4905$

Oklahoma

Dr. Fred Walker, Administrator

Oklahoma Cancer Registry - 0200

Oklahoma State Department of Health

1000 NE 10th Street

Oklahoma City OK 73117-1299

(405) $271-7353$

\section{Comments:}

Joined SEER Program in 1973. Also collects data on Native American residents of Arizona.

Ordering:

Data requests should be sent to Dr. Ben Hankey, SEER Program, $\mathrm{NCl}, 9000$ Rockville Pike, Bethesda MD 20892-4200; (301) 496-8510.

Comments:

Ordering:

\section{Comments:}

Strong research involvement with CCR. Good capability for geographic studies.

Ordering:

Submit request in writing. A review protocol is followed. Please call with questions.

\section{Comments:}

Ordering:

Comments:

Population-based for Cuyahoga County beginning in 1987. Covers Cuyahoga County, Lake Geauga County, parts of Medina County, Toledo, \& Massilon.

Ordering:

Comments:

Ordering: 
Oregon

Donald Austin, MD

Oregon Health Division

800 NE Oregon St., Suite 730

Portland OR 97232

(503) $731-4025$

Pennsylvania

Patricia W. Potrzebowski, Ph.D.

State Health Data Center

Commonwealth of Pennsylvania

Department of Health

P.O. Box 90, Room 127

Harrisburg PA 17108-9990

(717) $783-2548$

Rhode Island

Dr. John P. Fulton, Administrator

Rhode Island Cancer Registry

Rhode Island Department of Health

3 Capitol Hill

Providence RI 02908-5097

(401) 277-2553

Fax: (401) 277-1272

\section{South Carolina}

Margaret Senn

Dept. of Chronic Diseases

South Carolina Dept. of Health

2600 Bull Street

Columbia SC 29201

(803) $734-4790$

\section{South Dakota}

Everett Putnam, Statistician

South Dakota Department of Health

Pierre SD 57501-3182

(605) $773-3365$

\section{Tennessee}

Mr. George Wade

Tennessee Cancer Reporting System Department of Health and Environment 419 Cordell Hull Building

Nashville TN 37247.0360

(615) 741-0685

Fax: (615) 532-1886

\section{Texas}

Barry Wilson, Public Health Technician

Cancer Registry Division

Texas Department of Health

1100 West 49th Street

Austin TX 78756

(800) 252-8059

Fax: (512)-502-0862
Comments:

No central registry yet, but plans are underway to establish one soon. Largest hospital registry is Kaiser system, covering $20 \%$ of metropolitan area.

Ordering:

Comments:

Collects minor civil division codes (borough \& township).

Ordering:

Comments:

The Hospital Association of Rhode Island collects the cancer data \& uses the Rocky Mountain Cancer Data System.

Ordering:

The name of the principal investigator is required.

Comments:

Twelve hospital registries cover $60 \%$ of the cases. Pilot registry began in 1990 \& includes Charleston, Dorchester, \& Berkeley counties.

Ordering:

Comments:

Ordering:

Comments:

Ordering:

Data requests may need to be approved by the advisory committee.

\section{Comments:}

Data are complete for 5 regions for 1976-1985.

Mortality data available for entire state through 1991.

Ordering: 
Utah

William P. McWhorter, MD, MPH, Director

Utah Cancer Registry

Research Park

420 Chipeta Way, Suite 190

Salt Lake City UT 84108

(801) 581-8407

Fax: ((801) $581-4560$

Vermont

Leslie A. Weber, MS, Director

Vermont Cancer Registry

Division of Epidemiology \& Disease Prevention

Department of Health

P.O. Box 70

Burlington VT 05402

(802) 865-7749

Fax: (802) 863-7425

Virainia

M. Gillian Thompson, CTR, Director

Virginia Cancer Registry

State Health Department, Room 114

PO Box 2448

Richmond VA 23218

(804) 786-1668

Fax: (804) 786-1076

\section{Washington}

Joseph Castorina, MD, MPH

Department of Health

Health Promotion and Disease Prevention

PO Box 47835

Olympia WA 98504

(206) 586-7625

Fax: (206) $586-5440$

West Virginia

Loretta E. Haddy, MA, MS, State Epidemiologist

Surveillance and Disease Control

West Virginia Bureau of Public Health

1422 Washington Street, East

Charleston WV 25301

(304) 558-5358

Fax: (304) 558-6335

Wisconsin

Ms. Jerri Linn Phillips, MA, CTR

Wisconsin Cancer Reporting System

Department of Health \& Social Services

1 W. Wilson Street, Room 172, P.O. Box 309

Madison WI 53701-0309

(608) 266-0633

Fax: (608) 267-0352
Comments:

Part of the SEER Program.

Ordering:

Comments:

Surveyed hospital registries in 1990 . Statewide registry began in November, 1993. Uses CANSUR/NET software.

Ordering:

Comments:

Prior to 1989 , reporting was voluntary; $85 \%$ complete.

Ordering:

Comments:

Seattle area joined SEER Program in 1974; statewide reporting began in July, 1991. Ordering:

Comments:

Statewide collection of breast and cervical cancer cases only before 1992; all sites reportable beginning in 1993.

Ordering:

Send letter of request explaining funding and purpose of the study.

\section{Comments:}

Cancer has been reportable since 1933, but registry did not begin until 1976.

\section{Ordering:}

Send letter with request, explaining who is funding the study and what is the purpose of the study. Strict confidentiality requirements. 


\section{Wyoming}

Mary I. Seaver

Wyoming Central Tumor Registry

Division of Health and Medical Sciences

Hathaway Building, 4th floor

Cheyenne WY 82002

(307) $777-7958$

Fax: (307) 777-5402
Comments:

Uses the Rocky Mountain Cancer Data System.

Ordering:

Send letter with request explaining purpose of study. 


\section{Appendix C: Cancer Data Services}

\section{ACTUR}

Major J.A. Halvorson

Medical Policy Liaison Officer

Defense Medical Systems Support Center

5109 Leesburg Pike, Suite 917

Falls Church VA 22041-3201

(703) 756-0904

Fax: (703) 756-0971

\section{Cansur NET}

Mary Crist, CTR

Cansur NET

American College of Surgeons

55 East Erie Street

Chicago IL 60611

(312) 649-3920

Fax: (310) 649-4600

C/Net

Dr. Barry Gordon, Director

C/Net Project

California Public Health Foundation

2001 Addison St, Suite 210

Berkeley CA 94704

(510) 540-0778

\section{ELM Services/PREMIER Cancer Registry System}

\section{Dr. Lee Mortenson}

ELM Services/PREMIER Cancer Registry System

11600 Nebel St., Suite 201

Rockville MD 20852

(301) 984-1242

Fax: (301) 770-1949

\section{Medical Registry Services}

William Weiss, President

Medical Registry Services

One University Plaza

Hackensack NJ 07601

(201) 487-2266

Fax: (201 487-5184

\section{PC Dash}

Anna Marie Davidson

PC Dash

New Mexico Tumor Registry

900 Camino de Salud, N.E.

Albuquerque NM 87131-5306

(505) 277-5541

Fax: (505) 277-8572
Comments:

Registry of military cases since 1989. They are retrospectively collecting data for cases since 1979.

\section{Comments:}

Provides cancer data management software for facilities throughout the United States. Assists in data submission to state, central, and national organizations.

Comments:

Provides cancer registry software for hospital registries throughout the United States. Does not collect or store data, but assists central registry data collection.

\section{Comments:}

Provides data system services for hospitals which enter data on PC's. The data are uploaded to the ELM mainframe system. ELM's on-line, interactive aggregate National Oncology Data Base contains clinical \& demographic information on over 800,000 cases \& can be queried using Sybase or SPSS.

Comments:

Provides cancer registry software \& support for over 200 hospital registries nationwide.

\section{Comments:}

Data collection system for State of New Mexico \& Arizona Native Americans. Also the system is used by the Native American Health Service registry in Alaska. Reporting package includes summary and survival reports, plus cross-tabulations. 
Rocky Mountain Cancer Data System

\section{Larry Derrick}

Rocky Mountain Cancer Data Si'stem

420 Chipeta Way, Suite 120

Salt Lake City UT 84108

(801) $581-4307$

Fax (801) 581-5704

\section{TUMORS}

Gail Jolitz

The Upper Midwest Oncology Reg. System (TUMORS)

6500 Excelsior Blvd, PO Box 650

Minneapolis MN 55440

(612) $932-5140$

Fax (612) $932-5512$

\section{Comments:}

Provides services for the Walla Walla WA, Spokane WA, Cleveland OH, DC, AZ, CO, DE, ID, MT, NV, $\mathrm{RI}, \mathrm{UT}$, \& WY registries.

\section{Comments:}

Provides a support system to 23 hospital registries in MN, ND, \& SD. Hospital registries collect $\&$ store the data; a copy of data is maintained in aggregate database at TUMORS. 


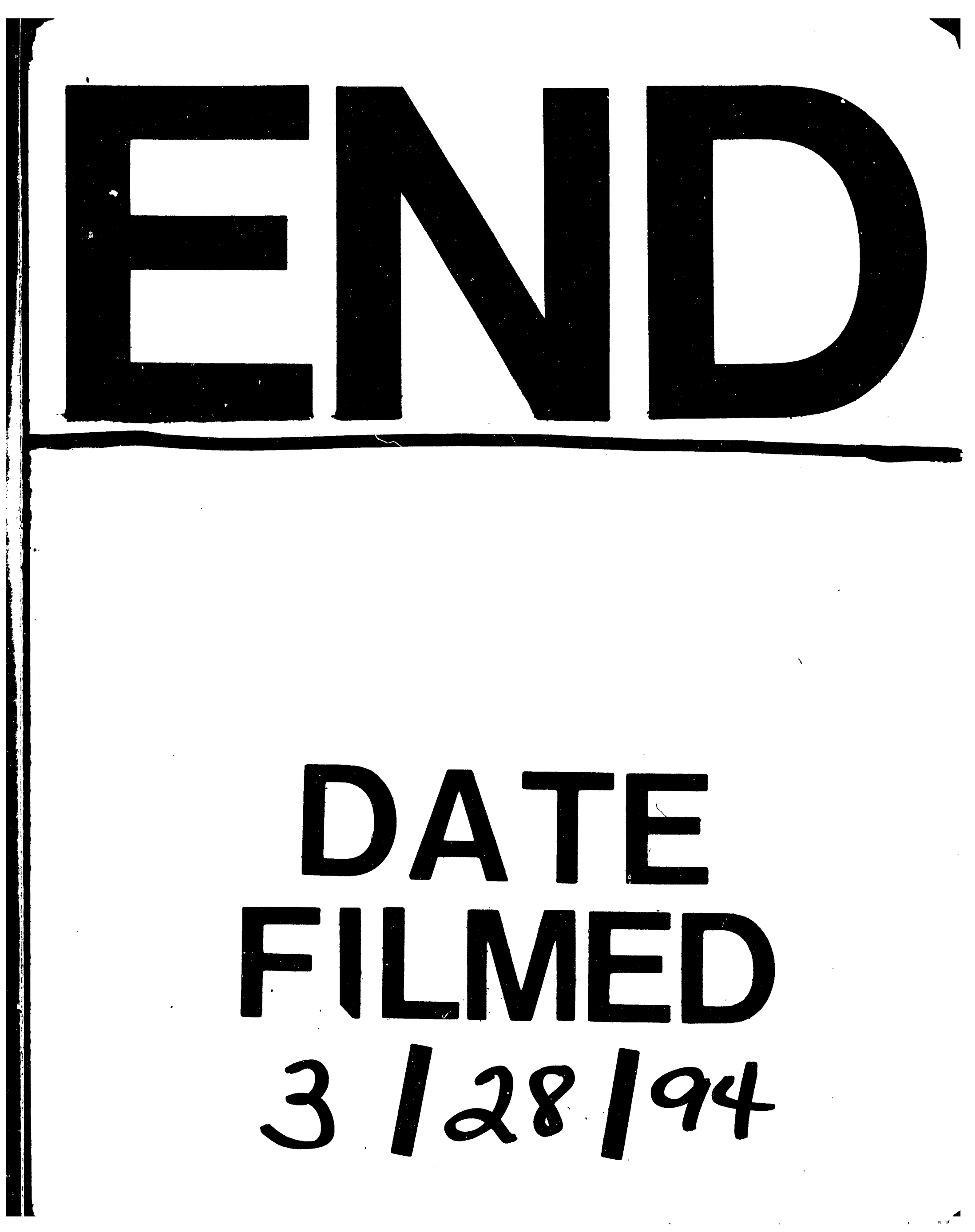


